\title{
Magnetic field measurements of Ap stars
}

\section{Discovery of a strong magnetic field in HD 18610}

\author{
Ch. Stütz ${ }^{1}$ T. Ryabchikova ${ }^{1,2}$, and W. W. Weiss ${ }^{1}$ \\ ${ }^{1}$ Institute for Astronomy (IfA), University of Vienna, Türkenschanzstrasse 17, 1180 Wien, Austria \\ 2 Institute of Astronomy, Russian Academy of Sciences, Pyatnitskaya 48, 109017 Moscow, Russia
}

Received 5 July 2002 / Accepted 12 February 2003

\begin{abstract}
HD 18610 was included in our project on abundance determination of rapidly oscillating (roAp) and non-oscillating (noAp) cool chemically peculiar stars. Already a preliminary investigation of the raw spectra indicated the presence of an intense magnetic field which was confirmed by a more detailed analysis.

To determine the magnetic field strength we primarily used the Fe II line at $6149.26 \AA$, a strong line which is split in a anomalous Zeeman doublet. HD 18610 is a Cr-Eu-Sr non-pulsating Ap star with a $T_{\text {eff }}$ and $\log g$ typical for roAp stars. The classification of this star as a CP2 star (Preston 1974) was confirmed with $\Delta$ a photometry by Maitzen \& Vogt (1983). In the present investigation we derived a mean magnetic field modulus of $|\boldsymbol{B}|=5700 \pm 200 \mathrm{G}$.
\end{abstract}

Key words. stars: individual: HD 18610 - stars: magnetic fields - stars: chemically peculiar

\section{Introduction}

The Ap star HD 18610 (CD-73 127) was observed within our program on the chemical analysis of CP star atmospheres for which more information can be found at http: //ams.astro.univie.ac.at

More than 30 rapidly oscillating Ap stars are known up to now, but so far only some of them have been spectroscopically investigated. The group of roAp stars provide an interesting potential for testing asteroseismic models, but unfortunately they are rather difficult to detect due to the small photometric amplitudes. Stars in the same parameter space as is occupied by roAp stars, but in which no pulsation has been detected (noAp stars) will help to investigate the physical background of pulsation. This led us to organize a dedicated spectroscopic survey for roAp and noAp stars. As a byproduct of our campaign we discovered the strong magnetic field of HD 18610. According to its photometric indices this star is situated in the temperature domain of roAp stars, but it shows no pulsation (Martinez \& Kurtz 1994).

\section{Observations}

Four spectra were obtained on August 27, 1999, by P. Martinez, P. Mittermayer and K. Zwinz with the Giraffe-EchelleSpectrograph of the South African Astronomical Observatory. An integration time of 1800 s per spectrum and a resolution of

Send offprint requests to: $\mathrm{Ch}$. Stütz,

e-mail: stuetz@tycho.astro.univie.ac.at

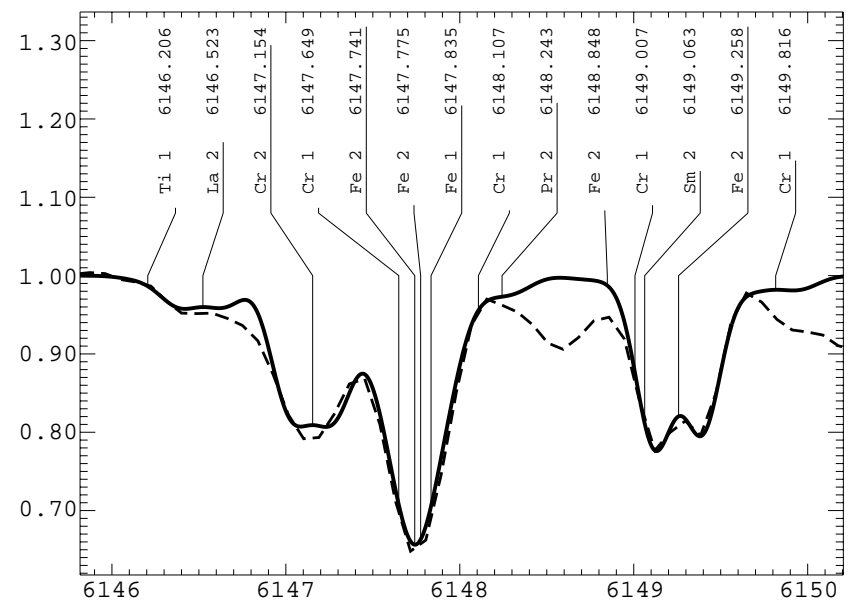

Fig. 1. A comparison between observations (thick dashed line) and synthetic spectrum (single line) in the region of two Fe II lines at 6147.74 A (blend of lines characterized by small effective Landé factors) and $6149.26 \AA$ (marginally blended line). The synthetic spectrum calculations were performed with a stratified $\mathrm{Fe}$ abundance and with a magnetic field of $5.7 \mathrm{kG}$. Note a resolved magnetic splitting of the second line.

$R=35000$ resulted in data with a combined signal to noise ratio of 130, sufficient for an abundance analysis. The observations have been reduced and flux normalized in Vienna using standard IRAF routines.

Already a first inspection of the spectra indicated the presence of a rather strong magnetic field which is reflected in partial magnetic splitting of a few spectral lines. In this context 


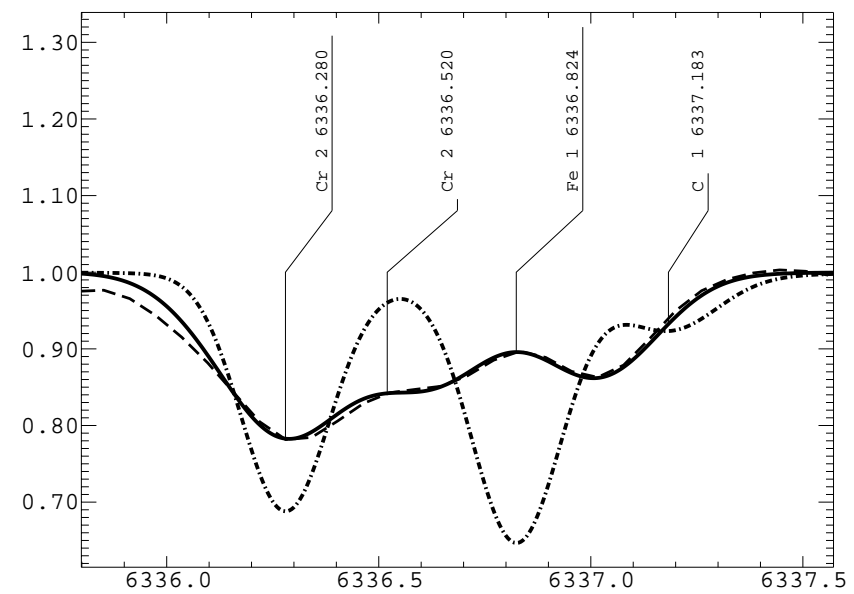

Fig. 2. Comparison between observed and calculated spectra in the region of the $\mathrm{Fe}_{\mathrm{I}}$ line at $6336.824 \AA\left(g_{\mathrm{eff}}=2.00\right)$. Thick dashed line observations. Dash-dotted line - the feature calculated without magnetic field. Single line - synthetic spectrum with a mean magnetic field modulus of $5.7 \mathrm{kG}$.

the Fe II line at $\lambda 6149.26 \AA$ is particularly prominent. The specific Zeeman pattern of this line (see Fig. 1) allows to reliably estimate the mean magnetic field modulus. The full Zeeman pattern of this line consists of two $\pi$ - and two $\sigma$-components with the same wavelength shift. For any magnetic field the line profile of this Fe II line in unpolarized light is represented by a simple doublet. $|\boldsymbol{B}|$ can be estimated according to

$\Delta \lambda_{\mathrm{Z}} / 2=g \cdot \lambda_{\mathrm{c}}^{2} \cdot|\boldsymbol{B}| \cdot 4.67 \times 10^{-13}$

where

$g\left(\hat{=} g_{\text {eff }}\right)=1.35$, effective Landé factor,

$\lambda_{\mathrm{c}}$, central wavelength of the unshifted line in $\AA$,

$\Delta \lambda_{\mathrm{Z}}$, measured Zeeman splitting in $\AA$,

$B$ in Gauss.

The Landé factors where taken from the Vienna Atomic Line Data Base (Piskunov et al. 1995; Kupka et al. 1999 and Ryabchikova et al. 1999). A first estimate of the magnetic field modulus was derived using Eq. (1). Wavelength separation between two components was estimated by fitting two gaussian to the observed line profiles. However, because of the rather low spectral resolution and partial blending of the processed lines (see Fig. 2), the accuracy of this measurement was not satisfying. An improvement can be achieved by computing synthetic spectra and fitting them to the observations by tuning the strength of the magnetic field used for the synthesis. All calculations were made with the syNTHMAG code written by N. Piskunov (1999). The atmospheric parameters, $T_{\text {eff }}=8100 \mathrm{~K}$ and $\log g=4.0$, were derived with starting points from the TEMPLOGG procedure (Rogers 1995) using Strömgren photometric indices extracted from the SIMBAD database. The best fit to the observed spectrum was obtained for a projected rotation velocity of $5 \mathrm{~km} \mathrm{~s}^{-1}$. After checking with other magnetically sensitive lines (see Fig. 2) we finally determined the value for the mean magnetic field modulus of HD 18610 to be $|\boldsymbol{B}|=5700 \pm 200 \mathrm{G}$.

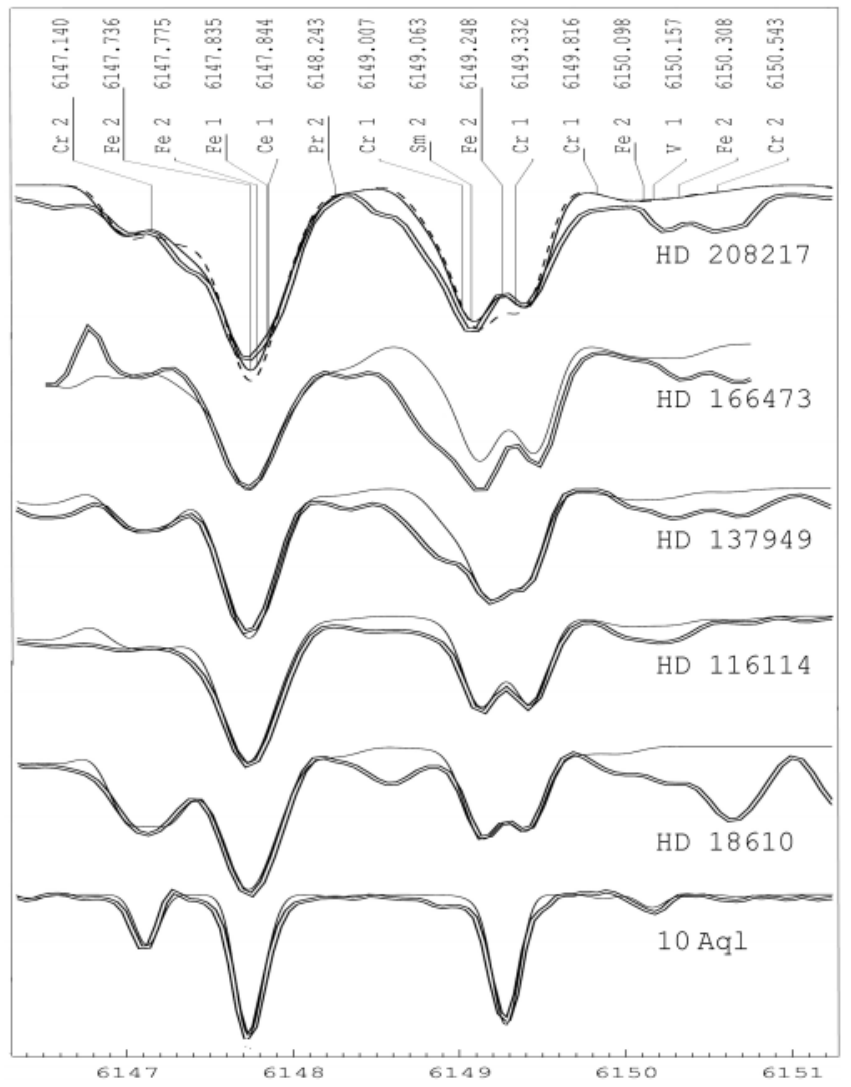

Fig. 3. The spectral region around $6148 \AA$ is shown for the stars of Table 1. Single line - synthetic spectrum; double line - observed spectrum. For the first star (HD 208217) two calculations are shown: the single line corresponds to $|\boldsymbol{B}|=7.3 \mathrm{kG}$, the dashed line to $|\boldsymbol{B}|=$ $6.5 \mathrm{kG}$. A spectrum of the star $10 \mathrm{Aql}$ (HD 176232), where no Zeeman splitting can be seen at our resolution, is plotted at the bottom of the plot.

\section{Discussion and conclusion}

For the stars listed in Table 1 in addition to HD 18610 we present only a first estimate for the mean magnetic field modulus based on Eq. (1). A comparison between magnetic synthetic calculations and observations is shown in Fig. 3. In this figure a strong blend with an unknown feature in the blue wing of Fe II $\lambda 6149.26 \AA$ line is seen in the spectra of some (mainly roAp) stars. This may influence the derived value of the magnetic field modulus. For a final analysis, our zero-order approximation of a radial magnetic field structure needs to be replaced by a more sophisticated model. Anyway, our estimates of the magnetic field modulus are in close agreement with measurements using the same Fe II line and spectra of much higher spectral resolution (Mathys et al. 1997). These measurements are also presented in Table 1.

For two stars, HD 116114 (BD -173829) and HD 137949 (BD164093), for which a rotation period higher than 5 years was proposed by Mathys et al. (1997), we obtained nearly the same values for the magnetic field strength. For HD 166473 (CD -37 12303) they observed a steady decrease of the magnetic field from $8550 \mathrm{G}$ in 1992 to $6400 \mathrm{G}$ in 1995 . Our spectrum of HD 208217 (CD -62 1372) corresponds to phase 0.08 
Table 1. Surface magnetic fields.

\begin{tabular}{lcccc}
\hline \hline Star & Type & Jul. Date & \multicolumn{2}{c}{$|\boldsymbol{B}|$ in Gauss } \\
& & & This paper & Mathys \\
\hline HD 18610 & noAp & 2451417.61 & 5700 & \\
HD 116114 & noAp & 2451416.23 & 6200 & 5953 \\
HD 137949 & roAp & 2451421.24 & 5000 & 4676 \\
HD 166473 & roAp & 2451417.27 & 7700 & $6400-8550$ \\
HD 208217 & noAp & 2451417.34 & 7300 & $6900-9000$ \\
\hline
\end{tabular}

of the proposed rotation period (see Mathys et al. 1997), with a minimum value of the magnetic field modulus 6879 Gauss.

Taking into account stratification and using a radial field geometry for the calculation of our synthetic spectra we discovered a mean magnetic field modulus of $|\boldsymbol{B}|=5700 \pm 200 \mathrm{G}$ for the noAp star HD 18610, which belongs to the group of $\mathrm{Cr}-$ $\mathrm{Eu}-\mathrm{Sr}$ chemically peculiar stars.

Acknowledgements. We want to thank U. Heiter and P. Mittermayer for their assistance at the reduction of the spectra of HD 18610, P. Knoglinger for providing us additional magnetic field measurements. This research was performed within the the working group "Asteroseismology along the Main Sequence" and was supported by the Austrian Fonds zur Förderung der wissenschaftlichen Forschung (project P14984) and the Jubiläumsfonds der Österreichischen Nationalbank. We also acknowledge using the SIMBAD data base.

\section{References}

Kochukhov, O., Landstreet, J. D., Ryabchikova, T., Weiss, W. W., \& Kupka, F. 2002, MNRAS, 337, L1

Kupka, F., Piskunov, N. E., Ryabchikova, T. A., Stempels, H. C., \& Weiss, W. W. 1999, A\&AS, 138, 119

Maitzen, H. M., \& Vogt, N. 1983, A\&A, 123, 48

Martinez, P., \& Kurtz, D. W. 1994, MNRAS, 271, 129

Mathys, G., Hubrig, S., Landstreet, J. D., Lanz, T., \& Manfroid, J. 1997, A\&AS, 123, 353

Piskunov, N. E., Kupka, F., Ryabchikova, T. A., Weiss, W. W., \& Jeffery, C. S. 1995, A\&AS, 112, 525

Piskunov, N. E. 1999, in 2nd Workshop on Solar Polarization, ed. J. Stenflo, \& K. N. Nagendra (Dordrecht: Kluwer Academic Publisher), 515

Preston, G. W. 1974, ARA\&A, 12, 257

Rogers, N. Y. 1995, Comm. Asteroseismol., No.78

Ryabchikova, T. A., Piskunov, N. E., Stempels, H. C., Kupka, F., \& Weiss W. W. 1999, Phys. Scr., T83, 162

Ryabchikova, T. A., Piskunov, N., Kochukhov, O., Tsymbal, V., Mittermayer, P., \& Weiss, W. W. 2002, A\&A, 384, 545 